\title{
Expression of Bcl-2, Smac and their correlation in endometriosis
}

\author{
Ping Zhu, Shuna Shen* \\ Department of Obstetrics and Gynecology, Baogang Hospital, Baotou, Inner Mongolia, China
}

Received: September 23, 2017

DOI: $10.14725 / d c c . v 4 n 4 p 11$

\author{
Accepted: November 6, 2017 Online Published: December 10, 2017 \\ URL: http://dx.doi.org/10.14725/dcc.v4n4p11
}

\begin{abstract}
Objective: To explore the expression of anti-apoptotic gene Bcl-2 and second mitochondria-derived activator of caspases (Smac) in endometriosis, the relationship of Bcl-2 and Smac with clinical staging and menstrual cycle, as well as correlation analysis. Methods: Thirty samples of endometrial tissues taken from endometriosis patients were chosen as the study group (endometriosis group), including 16 cases in the proliferative phase and 14 cases in the secretory phase. Thirty samples of normal endometrial tissues were chosen as the control group. Immunohistochemical method (SP) was used to determine the expression of Bcl-2 and Smac proteins in each group. Statistical analysis was carried out with SPSS 16.0 statistical package. The comparison of measurement data between two groups was made by use of chi-square $\left(\chi^{2}\right)$ test, and the correlation test of two indexes was performed by use of Spearman's correlation analysis. The difference in statistical data was of statistical significance $(p<.05)$. Results: The expression of Bcl-2 in eutopic and ectopic endometrial tissues in the endometriosis group was significantly higher than that in normal endometrial tissues in the control group, and the difference was statistically significant $(p<.05)$. The difference of Bcl-2 expression in all phases of both eutopic tissues in the endometriosis group and normal endometrial tissues was statistically significant $(p<.05)$, the positive expression in the secretory phase was obviously lower than that in the proliferative phase. The expression intensity of Smac in eutopic and ectopic endometrial tissues in the endometriosis group was lower than that in normal endometrial tissues in the control group, and the difference was of statistical significance $(p<.05)$. The expression intensity of Smac in ectopic endometrial tissues was lower than that in eutopic endometrial tissues, and the difference was of statistical significance $(p<.05)$; the expression intensity of Smac in the secretory phase was higher than that in the proliferative phase in normal endometrial tissues $(p<.05)$. The expression of Bcl-2 and Smac was not related to clinical staging of endometriosis $(p>.05)$. The expression of Bcl-2 was negatively correlated to the expression of $\operatorname{Smac}(p<.05)$.

Conclusions: The high expression of Bcl-2 and the low expression of Smac enhance the abilities of hyperplasia and antiapoptosis of ectopic endometrial cells, which leads to the occurrence and development of endometriosis.
\end{abstract}

Key Words: Endometriosis, Immunohistochemistry, Bcl-2, Smac

Endometriosis is a condition that endometrial tissues normally cover the inside of the uterus and outside of myometrium. This type of tissues can invade any part of the body, which is mostly commonly seen in ovaries. In recent years, it has been found that the sensitization of endometrial tissues to cell apoptosis is related to the progress of this disease. ${ }^{[1]}$

B-Cell leukemia/lymphoma 2 (Bcl-2 family) is a family of oncogenes, one of which firstly found to be related to cell viability is Bcl-2, ${ }^{[2]}$ an anti-apoptotic gene. Second mitochondria-derived activator of caspases (Smac), also

\footnotetext{
*Correspondence: Shuna Shen; E-mail: shunashen@126.com; Address: Department of Obstetrics and Gynecology, Baogang Hospital, Baotou, Inner Mongolia, China.
} 
known as direct IAP binding protein with low PI (DIA$\mathrm{BLO}$ ), is a recently discovered mitochondrial pro-apoptotic protein. ${ }^{[3]}$

In this study, immunohistochemical method was used to detect Bcl-2 and Smac, in order to study not only their expression in ectopic and eutopic endometrium in the endometriosis group and normal endometrium in the control group, but also the correlation between them. It was also intended to explore the significance of the above factors in the pathogenesis mechanism of endometriosis, to reveal the relationship between cell apoptosis disorder and the occurrence of the disease, provide early diagnosis and prognosis of endometriosis with more valuable information, and find a new theoretical basis for the non-surgical treatment of the disease.

\section{Data and methods}

\subsection{Research objects}

The research was made in three groups: endometriosis group (ectopic group and eutopic group) and normal endometrium group (control group).

\subsubsection{Ectopic group}

30 cases of patients ( 16 cases for the proliferative phase, 14 cases for the secretory phase), were given laparotomy operation or laparoscopic surgery due to endormetriosis (confirmed by postoperative pathological examinations) in Department of Obstetrics and Gynecology of Baogang Hospital from October 2010 to April 2012. According to clinical staging standards established by American Fertility Association (R-AFS) in 1985, 30 cases of patients, aged from 21 to 52 years, can be classified as follows: 14 cases for I-II, 16 cases for III-IV.

\subsubsection{Eutopic group}

Eutopic endometria were taken from the above endometriosis patients by diagnostic curettage or hysterectomy. Combined with the menstrual history, endometrial staging is based on the characteristics of endometrium morphology in different stages to make judgments.

\subsubsection{Control group}

30 cases of patients, randomly selected during the same period, were given hysterectomy due to cervical lesions without endometriosis and adenomyosis. Endometria taken from these patients were verified as normal endometrial tissues by postoperative pathological examinations.
All of the above subjects were of complete clinicopathological data and regular menstrual cycle, without oral steroid hormones taken in 6 months before surgery, with no IUD placed, no lesions in the endometrium and no history of endocrine diseases.

\subsubsection{Main reagents and experimental methods}

The main reagents were purchased from Wuhan Boster Biological Technology Co., Ltd. and Fuzhou Maixin Biotechnologies Development Company. Immunohistochemical method was used in strict accordance with kit instructions for staining.

\subsubsection{Image analysis}

The immunohistochemical SP method was used to carry out the experiment. In the image analysis, the average percentage of positive cells in each field was counted as the percentage of positive cells in the section, and then scored as follows: the percentage of positive cells more than $75 \%$ was 4, $51 \%-75 \%$ for $3,26 \%-50 \%$ for $2,6 \%-25 \%$ for 1 , less than $5 \%$ for 0 ; the positive intensity is based on the chromogenic reaction of most cells: dark brownish-yellow for 3 points; brownish-yellow for 2 points; light brownish-yellow for 1 point; no coloring or slight coloring for 0 point. The results were determined through comprehensive analysis: the product ( $>3$ points) obtained by multiplying the percentage of positive cells and the positive intensity together, can be used as the positive standard.

\subsubsection{Statistical analysis}

After the positive percentages of Bcl-2 and Smac proteins in the endometrium were measured, Excel 2000 was used to compute results and set up a database. SPSS 16.0 software package was applied to the statistical analysis. The comparison of measurement data between two groups was made by use of chi-square $\left(\chi^{2}\right)$ test, and the correlation test was performed by use of Spearman's correlation analysis. The difference in statistical data was of statistical significance $(p$ $<.05)$.

\section{Results}

\subsection{Expression of $\mathrm{Bcl}-2$}

The expression of Bcl-2 protein in the control group, eutopic group and ectopic group was of statistical difference $\left(\chi^{2}=\right.$ $17.104, p=.002)$. After examination, the expression of Bcl2 protein in the ectopic group was significantly higher than that in the control group. According to the statistical testing, 
the difference was statistically significant $\left(\chi^{2}=9.643, p<\right.$ $.05)$. The positive expression of Bcl-2 protein in the eutopic group was significantly higher than that in the control group. According to the statistical testing, the difference was statistically significant $\left(\chi^{2}=15.017, p<.05\right)$. According to the statistical testing, the difference of the positive expression of Bcl-2 protein in the ectopic group and the eutopic group was of no statistical significance $(p>.05)$. According to the statistical testing, the difference of the positive expression of Bcl-2 protein in the secretory phase and the proliferative phase in the ectopic group, was of no statistical significance $(p>.05)$; in the eutopic group and the control group, the positive expression of Bcl-2 protein in the secretory phase was dramatically lower than that in the proliferative phase, and the difference was of statistical significance $(p<.05$, see Tables 1-2 and Figure 1).

Table 1: The comparison of Bcl-2 expression in each group

\begin{tabular}{|c|c|c|c|c|c|}
\hline & \multirow{2}{*}{$\mathbf{n}$} & \multicolumn{2}{|c|}{+} & \multicolumn{2}{|c|}{-} \\
\hline & & $\mathbf{n}$ & $\%$ & $\mathbf{n}$ & $\%$ \\
\hline Control Group & 30 & 8 & 26.67 & 22 & 73.33 \\
\hline Eutopic Group & 30 & 23 & 76.67 & 7 & 23.33 \\
\hline Ectopic Group & 30 & 20 & 66.67 & 10 & 33.33 \\
\hline
\end{tabular}

Table 2: The comparison in Bcl-2 expression between the proliferative and the secretory phases in each group

\begin{tabular}{|c|c|c|c|c|c|c|c|}
\hline & Staging & $\bar{n}$ & $+(\mathbf{n})$ & $-(\mathbf{n})$ & Positive Rate (\%) & $\chi^{2}$ value & $p$ value \\
\hline \multirow{2}{*}{ Control Group } & Proliferative Phase & 16 & 7 & 9 & 43.75 & \multirow{2}{*}{5.117} & \multirow{2}{*}{$<.05$} \\
\hline & Secretory Phase & 14 & 1 & 13 & 7.14 & & \\
\hline \multirow{2}{*}{ Eutopic Group } & Proliferative Phase & 16 & 15 & 1 & 93.75 & \multirow{2}{*}{5.593} & \multirow{2}{*}{$<.05$} \\
\hline & Secretory Phase & 14 & 8 & 6 & 57.14 & & \\
\hline \multirow{2}{*}{ Ectopic Group } & Proliferative Phase & 16 & 11 & 5 & 68.75 & \multirow{2}{*}{0.067} & \multirow{2}{*}{$>.05$} \\
\hline & Secretory Phase & 14 & 9 & 5 & 64.29 & & \\
\hline
\end{tabular}
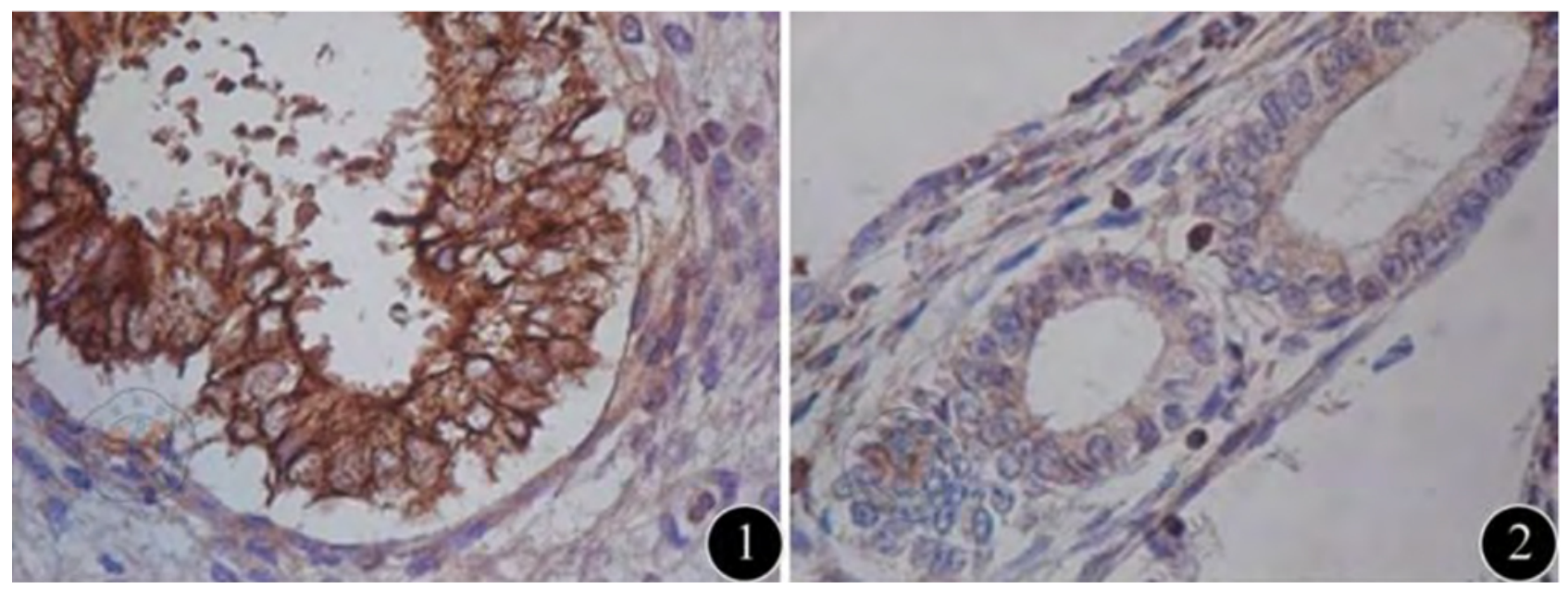

Figure 1: The expression of Bcl-2 (1) and Smac (2) in the ectopic group (Immunohistochemistry $\times 400$ )

\subsection{Expression of Smac}

The expression of Smac in the control group, eutopic group and ectopic group was of statistical difference $\left(\chi^{2}=19.530\right.$, $p=.000)$. After examination, the expression of Smac in the ectopic group was significantly lower than that in the

Published by New Century Science Press control group. According to the statistical testing, the difference was statistically significant $\left(\chi^{2}=19.461, p<.05\right)$. The positive expression of Smac in the eutopic group was significantly lower than that in the control group. According to the statistical testing, the difference was statistically 
significant $\left(\chi^{2}=5.554, p<.05\right)$. According to the statistical testing, the positive expression of Smac in the ectopic group and the eutopic group was of statistical significance $\left(\chi^{2}=\right.$ $5.079, p<.05$, see Table 3). The expression of Smac in the eutopic and the ectopic groups had no significant correlation to the expression in the menstrual cycle $(p>.05)$, while in the control group, the positive expression rate of Smac in the secretory phase was higher than that in the proliferative phase, and the difference was statistically significant $(p<$ .05 , see Table 4 and Figure 1).

Table 3: The comparison of Smac expression in each group

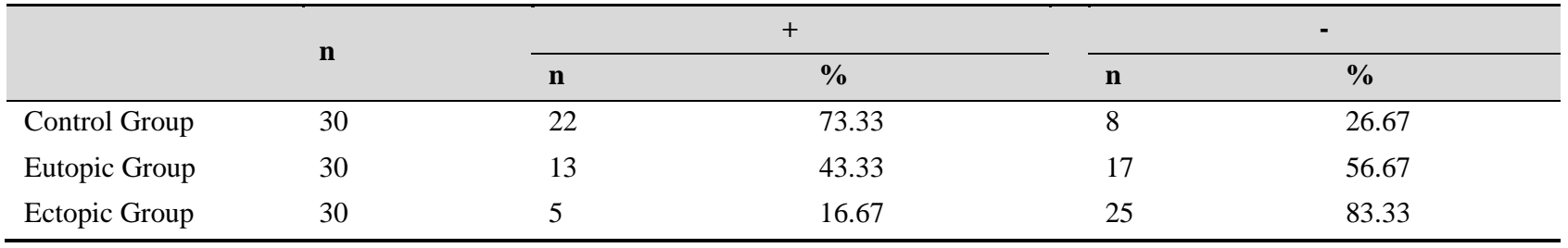

Table 4: The comparison in Smac expression between the proliferative and the secretory phases in each group

\begin{tabular}{|c|c|c|c|c|c|c|c|}
\hline & Staging & $\mathbf{n}$ & $+(\mathbf{n})$ & $-(\mathbf{n})$ & Positive Rate (\%) & $\chi^{2}$ value & $p$ value \\
\hline \multirow{2}{*}{ Control Group } & Proliferative Phase & 16 & 9 & 7 & 56.25 & \multirow{2}{*}{5.117} & \multirow{2}{*}{$<.05$} \\
\hline & Secretory Phase & 14 & 13 & 1 & 92.86 & & \\
\hline \multirow{2}{*}{ Eutopic Group } & Proliferative Phase & 16 & 7 & 9 & 43.75 & \multirow{2}{*}{0.002} & \multirow{2}{*}{$<.05$} \\
\hline & Secretory Phase & 14 & 6 & 8 & 42.86 & & \\
\hline \multirow{2}{*}{ Ectopic Group } & Proliferative Phase & 16 & 3 & 13 & 18.75 & \multirow{2}{*}{0.107} & \multirow{2}{*}{$>.05$} \\
\hline & Secretory Phase & 14 & 2 & 12 & 14.29 & & \\
\hline
\end{tabular}

Table 5: The comparison of Bcl-2 and Smac expression in different clinical stages of endometriosis [n, $(\%)]$

\begin{tabular}{lllllll}
\hline & $\mathbf{n}$ & \multicolumn{2}{c}{ Bcl-2 } & & \multicolumn{2}{c}{ Smac } \\
\cline { 3 - 4 } \cline { 6 - 7 } & & + & - & + & $6(42.86)$ & $8(57.14)$ \\
I-II & 14 & $11(78.57)$ & $3(21.43)$ & & $7(43.75)$ & $9(56.25)$ \\
\hline III-IV & 16 & $12(75.00)$ & $4(25.00)$ & & \\
\hline
\end{tabular}

\subsection{The relationship between the expression of $\mathrm{Bcl}-$ 2 and Smac and the clinical staging of en- dometriosis}

In the eutopic group, the difference of Bcl-2 expression in stage I-II and the stage III-IV was of no statistical significance $\left(\chi^{2}=0.053, p>.05\right)$. The difference of Smac expression in both stages was of no statistical significance $\left(\chi^{2}=\right.$ $0.002, p>.05$, see Table 5).

\subsection{The correlation of $\mathrm{Bcl}-2$ to Smac in en- dometriosis}

Spearman's correlation analysis showed that, in the eutopic group and the ectopic group, Bcl-2 was negatively correlated to Smac, and $r_{s}$ were $-0.933(p<.05)$ and $-0.875(p<$ $.05)$ respectively.

\section{Discussion}

\section{1 $\mathrm{Bcl}-2$ and endometriosis}

Bcl-2 family, is also known as B-cell leukemia/lymphoma 2 protein. Bcl-2 gene, a type of anti-apoptosis proteins obtained in 1984, is one of proto-oncogenes closely associated with apoptosis in the current discovery. ${ }^{[4]}$ During the process of apoptosis, Bcl-2 protein is activated and then translocated to the mitochondrial outer membrane, destroying its integrity, stimulating pro-apoptotic factors inside the mitochondria to be transformed and released, and promoting cell apoptosis. ${ }^{[5]}$ The mechanism of Bcl-2 in the inhibition of cell apoptosis is as follows: ${ }^{[6,7]}$ (1) Bcl-2 inhibits cytochrome $\mathrm{C}$ with the pro-apoptotic function being released from the mitochondria into the cytoplasm. The ions inside and outside the mitochondrial membranes reach equilibrium with each other, so that the ions in the mitochondrial matrix turn out to be hypertonic and inflated, resulting in mi- 
tochondrial outer membrane rupture, and then pro-apoptotic proteins are released from the intermembrane space into the cytoplasm. Pro-apoptotic proteins that enter the cytoplasm disrupt chromatins in the nucleus or activate Caspase factors, causing cell apoptosis consequently. (2) Bcl-2 can regulate cell transmembrane transport and affect the distribution of calcium ions inside and outside the cell membranes. Calcium ions can stimulate and activate endogenous endonucleases and gamma-glutamyl transpeptidase (GGT), thereby changing the role of the necleus in apoptosis. (3) Bcl-2 can be used as an antioxidant to change the oxidationreduction state of cells, further preventing components of cells from being destructed by redox.

The research results have indicated that the expression of $\mathrm{Bcl}-2$ protein in the normal endometrium is mainly in the cytoplasm, and the expression intensity in the secretory phase is lower than that in the proliferative phase. It is consistent with the theory that endometrial apoptosis is exacerbated in the secretory phase to promote the shedding of endometrium. The expression of Bcl-2 in the ectopic and the eutopic groups is higher than that in the control group, suggesting that $\mathrm{Bcl}-2$ might be involved in the occurrence and development of endometriosis and play a key role in it. It is consistent with the concept of "determinism of eutopic endometrium". Dmowski et al. ${ }^{[8]}$ used RT-PCR method to detect the expression of $\mathrm{Bcl}-2$ in the samples of ectopic endometrium, eutopic endometrium in endometriosis and normal endometrium. The results showed periodic changes in the expression of $\mathrm{Bcl}-2$ protein in the endometriosis eutopic endometrium and the normal endometrium, the expression of Bcl-2 in the secretory phase was significantly lower than that in the proliferative phase in any type of endometrium, and the expression in the eutopic endometrium was significantly higher than that in the normal endometrium during the secretory phase; high expression of $\mathrm{Bcl}-2$ protein in endometriosis ectopic endometrium showed no significant periodic changes. The experimental results supported this study.

\subsection{Smac and endometriosis}

Smac/DIABLO protein, i.e., second mitochondria-derived activator of caspases/direct IAP binding protein with low PI, isolated from HeLa cell experiments made in 2000, is a type of mitochondrial pro-apoptotic proteins involved in the downstream reaction of cell apoptosis. Human Smac gene is located on the long arm of chromosome 12, where 55 amino acids at the $\mathrm{N}$-terminus are called mitochondrial target sequence (MTS), the function of which is to ensure that unprocessed Smac proteins shall be located on the mitochondria, whereas MTS is decomposed by the mitochondrial signal peptide after Smac is transferred into the mitochondria. Eventually, umprocessed Smac proteins containing 184 amino acid residues are generated, with the apoptotic activity acquired simultaneously. ${ }^{[3,9]}$ The mechanism Published by New Century Science Press of Smac in the cell apoptosis is as follows: ${ }^{[10,11]}$ (1) Smac can directly interact with Caspase-9 to enhance cascade reactions of Caspases, increase the sensitivity of tumor cells to the apoptosis stimulation and promote cell apoptosis. (2) Smac can also enhance the activity of Caspase- 3 by promoting the proteolysis of pro-Caspase-3, while the activated Caspase- 3 in turn activates Caspase- 9 zymogens. Activated Caspase- 9 can also activate Caspase- 3 by trans-activation, forming positive feedback to promote apoptosis further. (3) During the research on the effect of chemotherapy on promoting tumor cell apoptosis, $\mathrm{Kim}^{[12]}$ found that Smac factor may promote cell apoptosis in a non-Caspase dependent way, which was supposed to be more efficient than the Caspase dependent way.

The results in this research showed that: the expression of Smac in the control group, the eutopic group and ectopic group was decreased significantly, the difference among the three groups was statistically significant $(p<.05)$, suggesting that Smac may participate in the occurrence and development of endometriosis, and play a key role in it. It is consistent with the concept of "determinism of eutopic endometrium". The expression of Smac in the eutopic and the ectopic groups had no significant correlation to the menstrual cycle and clinical staging $(p>.05)$, but in the control group, the expression of Smac was higher in the secretory phase than that in the proliferative phase $(p<.05)$; it is consistent with the theory that endometrial apoptosis is exacerbated in the secretory phase to promote the shedding of endometrium.

\subsection{Relationship between Bcl-2 and Smac}

The results of this study showed that Bcl-2 was negatively correlated to Smac, but the mechanism remained to be perfected; the synergistic effect of these two factors can lead to uncontrolled proliferation of endometrial cells in other parts outside the uterus, promote the decline in the proapoptotic ability of ectopic endometrial cells and the increase in the anti-apoptotic ability. Ectopic and eutopic endometrial apoptosis are disordered in patients with endometriosis, so that the apoptosis rate of endometrial cells is low with a high proliferation rate. Normal endometrial cells with biological activities flow upstream with menstruation into the abdominopelvic cavity, and these ectopic endometrial cells with low apoptosis rate will cause ectopic lesions to occur and develop where they are located. RNAi technology is a newly developed molecular biology technology. It can be combined with specific mRNA to disintegrate the target gene. The application of this technology silencing the specific gene provides a new development direction for the gene therapy of diseases.

\section{Conflicts of Interest Disclosure}

The authors have no conflicts of interest related to this article. 


\section{References}

[1] Johnson NP, Hummelshoj L. Consensus on current management of endometriosis. Hum Reprod. 2013; 28: 1552-1568. PMid: 23528916. https://doi.org/10.1093/humrep/det050

[2] Renault TT, Teijido O, Antonsson B, et al. Regulation of Bax mitochondrial localization by Bcl-2 and Bcl-x (L): keep your friends close but your enemies closer. Int J Biochem Cell Biol. 2013; 45(1): 64-67. PMid: 23064052. https://doi.org/10.1016/j . biocel.2012.09.022

[3] Bake V, Roesler S, Eckhardt I, et al. Synergistic interaction of Smac mimetic and IFN $\alpha$ to trigger apoptosis in acute myeloid leukemia cells. Cancer Letters. 2014; 35(52): 78-79. https://doi.org/ 10.1016/j.canlet. 2014.08.040

[4] Yang T, Zhang Y, Li Y, et al. High amounts of fluoride induce apoptosis/cell death in mature dameloblast-like LS8 cells by downregulating Bcl-2. Arch Oral Biol. 2013; 58(9): 1165-1173. PMid: 23598055. https://doi.org/10.1016/j. archoralbio.2013.03.016

[5] Liu ZK, Song T, Dou CW, et al. Detection of Bcl-2 in human hepatocellular carcinoma and down-regulation with siRNA. Chinese Journal of Cellular and Molecular Immunology. 2015; 31(2): 221-225. PMid: 25652864.

[6] Zhang HZ, Liu Y, Bai LJ, et al. Effect of proanthocyanidins on Bcl2 and Bax in rat retina after ischemia-reperfusion injury. Journal of Traditional Chinese Ophthalmology. 2013; 23(2): 83-86.
[7] Dinh C, Bas E, Dinh J, et al. Short interfering RNA against Bax attenuates tnfa-induced ototoxicity in rat organ of corti explants. Otolaryngol Head Neck Surg. 2013; 148: 834-840. PMid: 23401256. https : //doi .org/10.1177/0194599813477631

[8] Dmowski WP, Ding J, Shen J, et al. Apoptosis endometrial glandular and stromai cells in women with and without endometriosis. Hum Eprod. 2001; 16(9): 1802-1808. https://doi .org/10. 1093/humrep/16.9.1802

[9] $\mathrm{Hu}$ W, Wang F, Tang J, et al. Pro-apoptotic protein, Smac, mediates apoptosis in cisplatin-resistant ovarian cancer cells when treated with the anti-tumor agent, at101. J Biol Chem. 2012; 287(1): 6880. PMid: 22052903. https://doi.org/10.1074/jbc.M111. 271205

[10] Laukens B, Jennewein C, Schenk B, et al. Smac Mimetic Bypasses Apoptosis Resistance in FADD- or Caspase-8-Deficient Cells by Priming for Tumor Necrosis Factor $\alpha$-Induced Necroptosis. Neoplasia. 2011; 13(10): 370-371. https://doi.org/10.1593/neo. 11610

[11] Su BC, Mo FE. CCN1 enables Fas ligand-induced apoptosis in cardiomyoblast $\mathrm{H} 9 \mathrm{c} 2$ cells by disrupting caspase inhibitor XIAP. Cellular Signaling. 2014; 5(13): 498-499. https ://doi .org/10. 1016/j.cellsig. 2014.02.019

[12] Kim R. Recent advances in understanding the cell death pathways activated by anticancer therapy. Cancer. 2005; 103(8): 1551. PMid: 15742333. https ://doi.org/10.1002/cncr. 20947 